\title{
Identification of a novel $A R L 13 B$ variant in a Joubert syndrome-affected patient with retinal impairment and obesity
}

\author{
Sophie Thomas ${ }^{1,2}$, Vincent Cantagrel ${ }^{1,2,3}$, Laura Mariani ${ }^{4}$, Valérie Serre ${ }^{1,5}$, Ji-Eun Lee ${ }^{3}$, Nadia Elkhartoufi ${ }^{1,2,6}$, \\ Pascale de Lonlay ${ }^{1,2,6}$, Isabelle Desguerre ${ }^{7}$, Arnold Munnich ${ }^{1,2,6}$, Nathalie Boddaert $^{8}$, Stanislas Lyonnet ${ }^{1,2,6}$, \\ Michel Vekemans ${ }^{1,2,6}$, Steven N Lisgo ${ }^{9}$, Tamara Caspary ${ }^{4}$, Joseph Gleeson ${ }^{3}$ and Tania Attié-Bitach ${ }^{\star, 1,2,6}$
}

Joubert syndrome (JS) is a genetically heterogeneous autosomal recessive ciliopathy with 22 genes implicated to date, including a small, ciliary GTPase, ARL13B. ARL13B is required for cilia formation in vertebrates. JS patients display multiple symptoms characterized by ataxia due to the cerebellar vermis hypoplasia, and that can also include ocular abnormalities, renal cysts, liver fibrosis or polydactyly. These symptoms are shared with other ciliopathies, some of which display additional phenotypes, such as obesity. Here we identified a novel homozygous missense variant in ARL13B/JBTS 8 in a JS patient who displayed retinal defects and obesity. We demonstrate the variant disrupts ARL13B function, as its expression did not rescue the mutant phenotype either in Arl13 $b^{\text {scorpion }}$ zebrafish or in Arl13bennin mouse embryonic fibroblasts, while the wild-type $A R L 13 B$ did. Finally, we show that ARL13B is localized within the primary cilia of neonatal mouse hypothalamic neurons consistent with the known link between hypothalamic ciliary function and obesity. Thus our data identify a novel $A R L 13 B$ variant that causes JS and retinopathy and suggest an extension of the phenotypic spectrum of $A R L 13 B$ mutations to obesity. European Journal of Human Genetics (2015) 23, 621-627; doi:10.1038/ejhg.2014.156; published online 20 August 2014

Joubert syndrome (JS [MIM 213300]) is a rare genetically heterogeneous inherited disorder. JS is characterized by congenital ataxia, hypotonia, developmental delay and at least one of the following features: neonatal respiratory disturbances and abnormal eye movements, including nystagmus and oculomotor apraxia. ${ }^{1}$ The neuroradiological hallmark in JS is a peculiar malformation of the midbrain-hindbrain junction known as the 'molar tooth sign', consisting of cerebellar vermis hypoplasia or dysplasia, thick and horizontally oriented superior cerebellar peduncles, and an abnormally deep interpeduncular fossa. In addition, extraneurological signs such as retinal abnormalities, renal cysts, hepatic fibrosis or polydactyly can be observed in Joubert syndrome and related disorders (JSRD). ${ }^{2}$

Twenty-two causative JS genes have been identified to date, all encoding proteins localized within or near the primary cilium, thus including JS in the group of ciliopathies. Primary cilia are known to play key roles in the development and functioning of several cell types, including retinal photoreceptors, neurons, and the epithelial cells comprising kidney tubules and bile ducts. ${ }^{3}$ In the developing cerebellum and brainstem, primary cilia regulate major signal transduction pathways, and have been implicated in both neuronal cell proliferation and axonal migration. ${ }^{4}$ In particular, primary cilia are required for Sonic Hedgehog $(\mathrm{SHH})$ signaling and for $\mathrm{SHH}-$ dependent cerebellar development, ${ }^{5-7}$ including in humans. ${ }^{8}$

Here we report a novel homozygous missense $A R L 13 B$ variant in a consanguineous Joubert patient from Tunisia with retinal involvement and obesity. Three ARL13B variants (R79C, R200C and W82X) were previously reported in two families with JS associated or not with retinal anomalies. ${ }^{9}$ ARL13B is a member of the ADP-ribosylation factor-like (ARL) family of small GTPases of the RAS superfamily. In model organisms, variants in $A R L 13 B$ have been linked to cilia assembly and kidney cyst formation. In zebrafish, analysis of the cystic kidney variant arl13b $b^{\text {scorpion }(s c o)}$ showed that ARL13B is required for cilia formation in the kidney duct. ${ }^{10}$ In mouse, loss of Arl13b function results in disrupted cilia and defective SHH signaling due to the involvement of ARL13B in the movement of $\mathrm{SHH}$ signaling components in and out of the cilia. ${ }^{11,12}$ Similarly, arl-13, the Caenorhabditis elegans orthologue of ARL13B, functions in ciliary membranes, where it is involved in ciliary transmembrane protein localization and transport of proteins to the tip of the cilium. ${ }^{13,14}$ More recently, Arl13b signaling in primary cilia has been shown to be crucial for the polarization of radial glial scaffold, an essential step in cerebral cortex formation. 15

To validate the effect of this novel variant in vivo, we took advantage of the phenotypes observed in zebrafish $\operatorname{arl} 13 b^{s c o}$ mutants and in Arl13b $b^{h n n}$ mouse embryonic fibroblasts (MEFs) and tested the ability of mutated human $A R L 13 B$ to rescue these phenotypes. We found that wild-type, but not mutant, $A R L 13 B$ is able to rescue those phenotypes, suggesting that the variant we identified is pathogenic. This JS patient also presented with obesity, which is a common feature of some ciliopathies such as Bardet-Biedl (BBS, MIM 209900),

${ }^{1}$ INSERM U1163, Hôpital Necker-Enfants Malades, Paris, France; ${ }^{2}$ Université Paris Descartes, Sorbonne Paris Cité, Institut Imagine, Paris, France; ${ }^{3}$ Laboratory of Neurogenetics, Howard Hughes Medical Institute, Department of Neurosciences, University of California, San Diego, La Jolla, CA, USA; ${ }^{4}$ Department of Human Genetics, Emory University School of Medicine, Atlanta, GA, USA; ${ }^{5}$ UMR7592CNRS, Jacques Monod Institute, Paris Diderot University, Paris, France; ${ }^{6}$ Département de Génétique, Hôpital Necker-Enfants Malades, AP-HP, Paris, France; ${ }^{7}$ Service de neurométabolisme, Hôpital Necker-Enfants Malades, AP-HP, Paris, France; ${ }^{8}$ Radiologie Pédiatrique et INSERM U-797, Hôpital Necker-Enfants Malades, AP-HP, Paris, France; ${ }^{9}$ The MRC-Wellcome Trust Human Developmental Biology Resource (HDBR), Institute of Genetic Medicine, International Centre for Life, Central Parkway, Newcastle Upon Tyne, UK

*Correspondence: Professor T Attie-Bitach, Departement de Genetique et INSERM U1163, Hopital Necker-Enfants Malades, 149 rue de Sèvres, Paris 75015, France. Tel: + 331 44495144; Fax: + 331 44495150; E-mail: tania.attie@inserm.fr

Received 10 January 2014; revised 27 June 2014; accepted 9 July 2014; published online 20 August 2014 
Alström (ALS, MIM 203800) and MORM (mental retardationobesity-retinopathy-micropenis, MIM 610156) but is rarely observed in JS. Recent evidence in ciliopathies has linked obesity to the regulation of homeostasis within the hypothalamus. Consistent with this, we found ARL13B localization within the primary cilia of hypothalamic neurons.

\section{MATERIALS AND METHODS \\ Patient}

The JS sib we studied is born to first-cousin parents from Tunisia. The affected boy corresponds to patient 3 already reported by Romano et al. ${ }^{16}$ Pregnancy was uneventful, and the boy was born at 38 gestation weeks, with normal birth weight $(3650 \mathrm{~g})$ and height $(51 \mathrm{~cm})$, but head circumference $(\mathrm{HC})$ at $+3 \mathrm{ds}$ $(37 \mathrm{~cm})$. He was referred to our hospital at 1 month of age for abnormal eye movements which was present since birth and disappearing during sleep and breastfeeding. At clinical examination, $\mathrm{HC}$ was at $+3 \mathrm{ds}(40 \mathrm{~cm})$, and persistent jaundice, hepatomegaly, hyperventilation access, pyramidal hypertonia and the absence of ocular contact were noted. At 4 months, jaundice and hepatomegaly had disappeared and a first brain MRI was described as normal. At 13 months, a psychomotor delay was noted and a retinopathy was suspected upon fundus examination showing optic disc pallor, confirmed by an extinguished electroretinogram. An overweight was noted $(13 \mathrm{~kg},+2 \mathrm{ds})$. At 16 months, a complete metabolic screening was performed, as well as heart and abdominal ultrasounds and skeletal $\mathrm{X}$ rays that revealed no anomalies. An electromyogram was also normal, and several electroencephalograms revealed no paroxystic anomalies, but a global low pattern. Ataxia was noted at 2 and 5 years. He walked at 3 years but had no speech, and weight and $\mathrm{HC}$ were consistently at +2 and $+3 \mathrm{ds}$, respectively. The diagnosis of JS was confirmed by reviewing the brain MRI performed at 16 months, which showed a molar tooth sign without supratentorial anomalies.

Informed consent for molecular analysis was obtained and the study was approved by the ethical committee of Paris Ile de France II.

\section{Genome linkage screening and linkage analysis}

Genome-wide homozygosity mapping was performed using 250K Affymetrix single-nucleotide polymorphism (SNP) arrays. Data were evaluated by calculating multipoint lod scores across the whole genome using MERLIN software (http://www.sph.umich.edu/csg/abecasis/Merlin), assuming recessive inheritance with complete penetrance.

\section{Sequencing of $A R L 13 B$}

Genomic DNA was extracted from peripheral blood samples. Primers were designed in introns flanking the 10 exons using the 'Primer 3' program (http://fokker.wi.mit.edu/primer3/input.htm) according to reference sequence NM_182896.2 and are listed in Supplementary Table 1. PCR was performed with a touchdown protocol consisting of denaturation for $30 \mathrm{~s}$ at $96^{\circ} \mathrm{C}$, annealing for $30 \mathrm{~s}$ at a temperature ranging from 64 to $50^{\circ} \mathrm{C}$ (decreasing by $1{ }^{\circ} \mathrm{C}$ during 14 cycles, then 20 cycles at $50^{\circ} \mathrm{C}$ ) and extension at $72{ }^{\circ} \mathrm{C}$ for $30 \mathrm{~s}$. PCR products were treated with ExoSAP-IT (USB Corp., Cleveland, OH, USA), and both strands were sequenced with the appropriate primer and the 'BigDye' terminator cycle sequencing kit (Applied Biosystems Inc., Foster City, CA, USA) and analyzed on ABI3130 automated sequencers. The DNA variant numbering system we used is based on cDNA sequence with +1 corresponding to the A of the ATG translation initiation codon in the reference sequence. The ARL13B variant reported has been submitted to the LOVDdatabase (http://databases.lovd.nl/shared/genes/ARL13B; Individual ID: 00017612).

\section{Bioinformatics}

The three-dimensional structure of the human ARL13B (residues 20-217) was modeled by comparative protein modeling methods and energy minimization, using the Swiss-Model program in the automated mode. ${ }^{8,17,18}$ The $2.5 \AA$ coordinate set for the CrArl13b (pdb code: 4M9Q) was used as a template for modeling the human ARL13B protein. Swiss-Pdb Viewer 3.7 (http://www.expasy.org/spdbv) was used to analyze the structural insight into ARL13B variant and visualize the structures.

\section{Zebrafish characterization}

The $\operatorname{arl} 13 b^{s c o /+}$ line was obtained from the Zebrafish International Resource Center (ZIRC) under an approved animal protocol at UCSD. One-cell and two-cell stage embryos from arl $13 b^{s c o /+}$ matings were injected with $50 \mathrm{pg}$ in vitro transcribed capped open reading frame RNA (Ambion Message Kit) of human wild-type or mutant transcript. After $72 \mathrm{~h}$, embryos were phenotyped by an investigator who was blinded to the genotype, and then all embryos were subject to genotyping for correlation.

\section{Arl13b transfections in mouse embryonic fibroblasts}

Arl13b protein-null (Arl13b $b^{h n n / h n n}$ ) mouse embryonic fibroblasts (MEFs) were plated on glass coverslips coated with $0.1 \%$ gelatin in six-well plates and cultured in DMEM supplemented with 10\% FBS and 1\% penicillin/streptomycin. When MEFs were $\sim 50 \%$ confluent, each well was co-transfected with $2.5 \mu \mathrm{g}$ of a ubiquitous DsRed expression construct and $2.5 \mu \mathrm{g}$ of either wildtype or mutant Arl13b expression construct. Transfections were performed using Lipofectamine-2000 (Invitrogen, Carlsbad, CA, USA; Cat. \#11668) according to the manufacturer's protocol. After transfection, the Arl13b $b^{h n n / h n n}$ MEFs were cultured in serum-free DMEM for $24 \mathrm{~h}$ to induce cilia formation. MEFs were fixed in $4 \%$ paraformaldehyde, blocked and permeabilized in antibody wash solution (PBS with $1 \%$ heat-inactivated normal goat serum and $0.1 \%$ Triton-X 100), and stained with antibodies against Arl13b (rabbit polyclonal, T. Caspary, 1:1500) and DsRed (rat monoclonal, Chromotek, Cat. \#5f8, 1:1000). Secondary antibodies used were Alexa Fluor 488 goat anti-rabbit (Invitrogen, Cat. \#11008) and Alexa Fluor 594 chicken anti-rat (Invitrogen, Cat. \#21471), both at 1:500, and Höechst nuclear marker (1:3000). Stained coverslips were mounted in Pro-Long Gold anti-fade reagent (Invitrogen, Cat. \#P36930) and imaged with a Leica CTR6000 fluorescent microscope using SimplePCI microscopy software (Hamamatsu Corporation, Sewickley, PA, USA). To prevent experimenter bias, transfected MEFs were identified via DsRed staining before imaging in the green channel to detect Arl13b. After imaging, the total number of transfected MEFs (defined by the presence of DsRed) was compared to the number of transfected MEFs exhibiting rescue (defined by the presence of a normal, Arl13b-positive cilium) to determine the percentage rescue in wild-type and mutant transfected conditions. This experiment was repeated in triplicate to calculate the mean percentage rescue in wild-type and mutant transfected conditions. A two-tailed Student's $t$-test was used to find the $P$-value for the difference in means.

\section{In situ hybridization}

Human embryos were obtained from the MRC/Wellcome-Trust-funded Human Developmental Biology Resource (HBDR, http://www.hdbr.org), with appropriate maternal written consent and appropriate ethical approval. The HDBR is regulated by the UK Human Tissue Authority (HTA; www.hta.gov. uk) and operates in accordance with the relevant HTA Codes of Practice.

Embryos were staged using external anatomical reference features ${ }^{19}$ and fixed overnight at $4{ }^{\circ} \mathrm{C}$ in $0.1 \mathrm{M}$ phosphate buffered saline (PBS) containing $4 \%$ paraformaldehyde. Embryos were then paraffin wax embedded and microtome sectioned. The in situ hybridization was performed using Digoxygen-labelled riboprobes in vitro transcribed from a linearized pCFRbluntII-TOPO vector containing the insert sequence NM_182896.1 nc 5021411 using SP6 (antisense) and T7 (sense) RNA polymerases. The riboprobes were annealed to target mRNA for $16 \mathrm{~h}$ at $68^{\circ} \mathrm{C}$ using previously described methods. ${ }^{20}$

\section{Immunofluorescence experiments}

IF and imaging were performed as previously described. ${ }^{9}$

\section{RESULTS}

We studied a consanguineous Tunisian family with one affected sib presenting with JS and associated features, including hypotonia, ataxia, breathing anomalies, oculomotor apraxia, abnormal eye 
movements, severe developmental delay and obesity (patient 3 already reported by Romano et al., ${ }^{16}$ pedigree of the family in Figure 1a). The JS diagnosis was confirmed with a brain MRI, which showed a molar tooth sign without supratentorial anomalies (Figure $1 \mathrm{~b}-\mathrm{d}$ ). Upon fundus examination optic disc pallor was observed and the boy's electroretinogram was extinguished. ${ }^{16}$ Through mapping at known JBS loci, we found homozygosity at the ARL13B locus. We direct sequenced the 10 coding exons in the patient and identified a novel homozygous missense variant: c.257A $>$ G, p.Tyr86Cys (hereafter, Y86C). This variant was absent from 240 Tunisian control chromosomes, as well as from dbSNP, 1000 Genome and EVS databases (http://evs.gs.washington. edu/EVS/). Parental DNA was not available for segregation analysis.

To further understand the effect of the Y86C variant on ARL13B function at the molecular level, we undertook in silico analysis. Amino-acid sequence alignment for ARL13B from nine different species showed that Y86 has been conserved in ARL13B since our common ancestor with C. elegans (Figure 2a) and is also conserved across many other proteins in the ARF family. The Y86C variant was predicted to affect protein function by PolyPhen-2 (http://genetics. bwh.harvard.edu/pph2/), SIFT (http://sift.jcvi.org/) and Mutation
Taster (http://alamut.interactive-biosoftware.com/tmp/mutation_taster_ form_JMg26.html). Y86 is not predicted to be phosphorylated according to the phosphorylation prediction software, Scansite (http://scansite.mit.edu/), which works on a system that incorporates sequence conservation and surface accessibility data with the predictions of NetPhos (http://www.cbs.dtu.dk/services/NetPhos/) and Scansite webtools. To study how the Y86C variant may affect ARL13B function we used the recently crystallized $\mathrm{CrArl}_{13 \mathrm{~B}^{21}}$ as template to model the human amino-acid sequence of ARL13B. Even if Y86 is not conserved in C.reinhardtii (Figure 2a), this is the only ARL13B structure published to date. In contrast to the previously identified $A R L 13 B$ variant at R79, which normally establishes a hydrogen bond with D30 from the GTP binding domain, ${ }^{9}$ Y86 appears not directly involved in GTP binding but in the switch II region (Figure $2 \mathrm{~b}$ ). In addition, we have identified two other aromatic residues: H117 and W82 located $<5.5 \AA$ from the Y86, contributing to Van der Walls forces stabilizing this region of the protein. The Y86C variant is predicted to destabilize these Van der Waals interactions, probably enhancing a local flexibility and thus altering the functional properties of the Switch II region. a

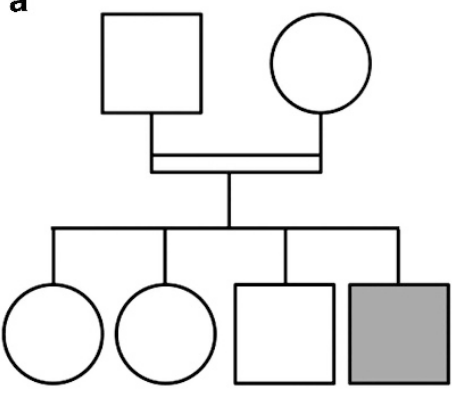

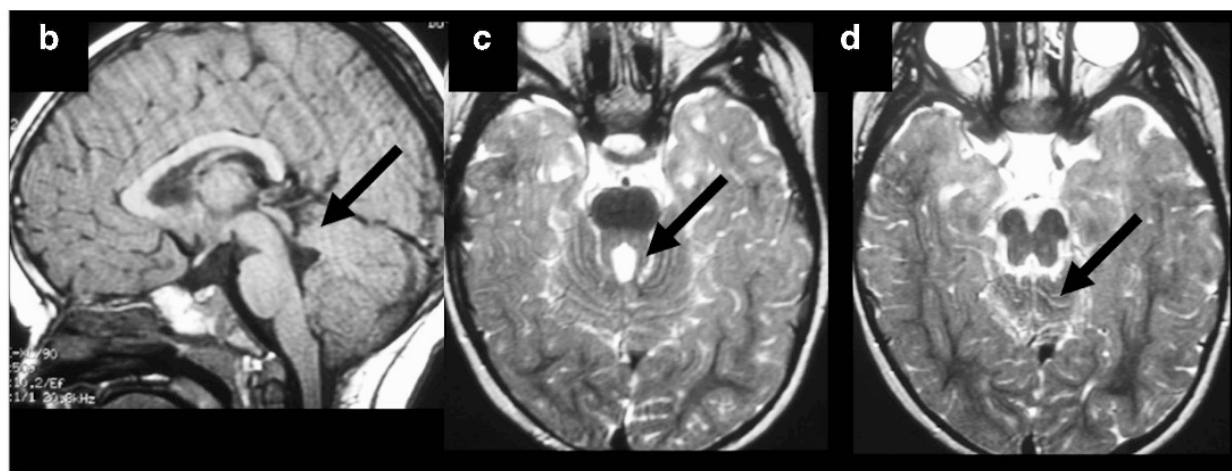

Figure 1 Pedigree of the studied family and brain MRI of the affected boy at 16 months. (a) Pedigree of the reported consanguineous Tunisian family with three healthy children and one boy presenting with JS, retinal anomalies and obesity. (b) Sagittal T1-weighted image shows in the affected patient a superior vermian dysgenesis (arrow) with hypoplastic or agenetic middle and inferior segments of the vermis. (c, d) Axial T2 FSE-weighted images demonstrate the abnormally thickened and elongated superior cerebellar peduncles and the molar tooth sign in the affected patient (c, arrow), as well as the superior vermian dysgenesis (d, arrow).

a

\begin{tabular}{|c|c|}
\hline H.sapiens & FEVTIFDLGGGIRIRGIWKNYYAESYGVIFVVDSSDEERMEETKE \\
\hline taurus & FEVTIFDLGGGKRIRGIWKNYY|AESYGVIFVVDSSDEERMEETKE \\
\hline norvegicus & FEVTIFDLGGGKRIRGIWKNYYAESYGVIFVVDSSDEDRMEETKE \\
\hline gallus & FEVTIFDLGGGKRIRNIWRNYYAESYGVIFVVDSSDIERMEETKE \\
\hline tropicalis & FDITMFDLGGGKRIRGIWKNYY \\
\hline rerio & YYSESYGVVFVVDSSDVQRIQETF \\
\hline elegans & IYYAEVHGI IYVIDYSTDETFTES \\
\hline nhardtii & AEVHAIVYVVDAADPGRFEE \\
\hline
\end{tabular}

109

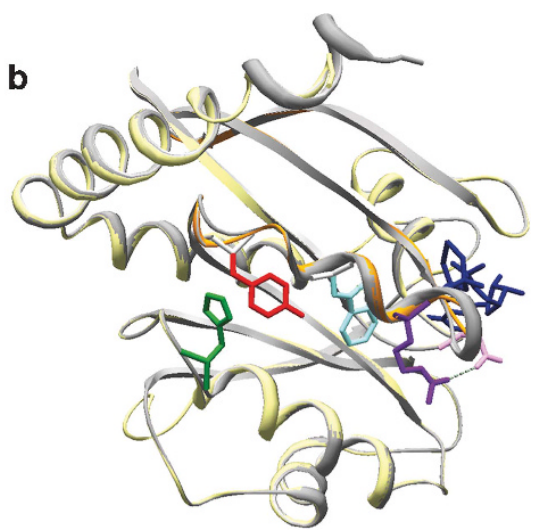

Figure 2 Alignment and conservation of Y86 residue (red box). Amino-acid sequences for ARL13B from 9 different species have been aligned showing high conservation of $Y 86$ residue (a). Homology modeling of ARL13B protein (yellow) using the crystal structure of crArl13B as a template (PDB code 4M9Q): 21 crArl13b (grey) is co-cristallized with phosphoaminophosphonic acid-guanylate ester (GNP; dark blue), magnesium and sulfate ions. Unlike R79 (light purple), which normally establishes a hydrogen bond (kaki dashed) with D30 (pink) from the GTP binding domain (Cantagrel et al. ${ }^{9}$ ), Y86 (red) appears not directly involved in GTP binding but is localized in the switch II region (orange) (b). Aromatic residues H117 and W82 are located $<5.5 \AA$ from Y86 $(4,01 \AA$ and 5.39 respectively) suggesting that the Van der Waals interactions between those 3 aromatic residues should be destabilized by the Y86C substitution. 

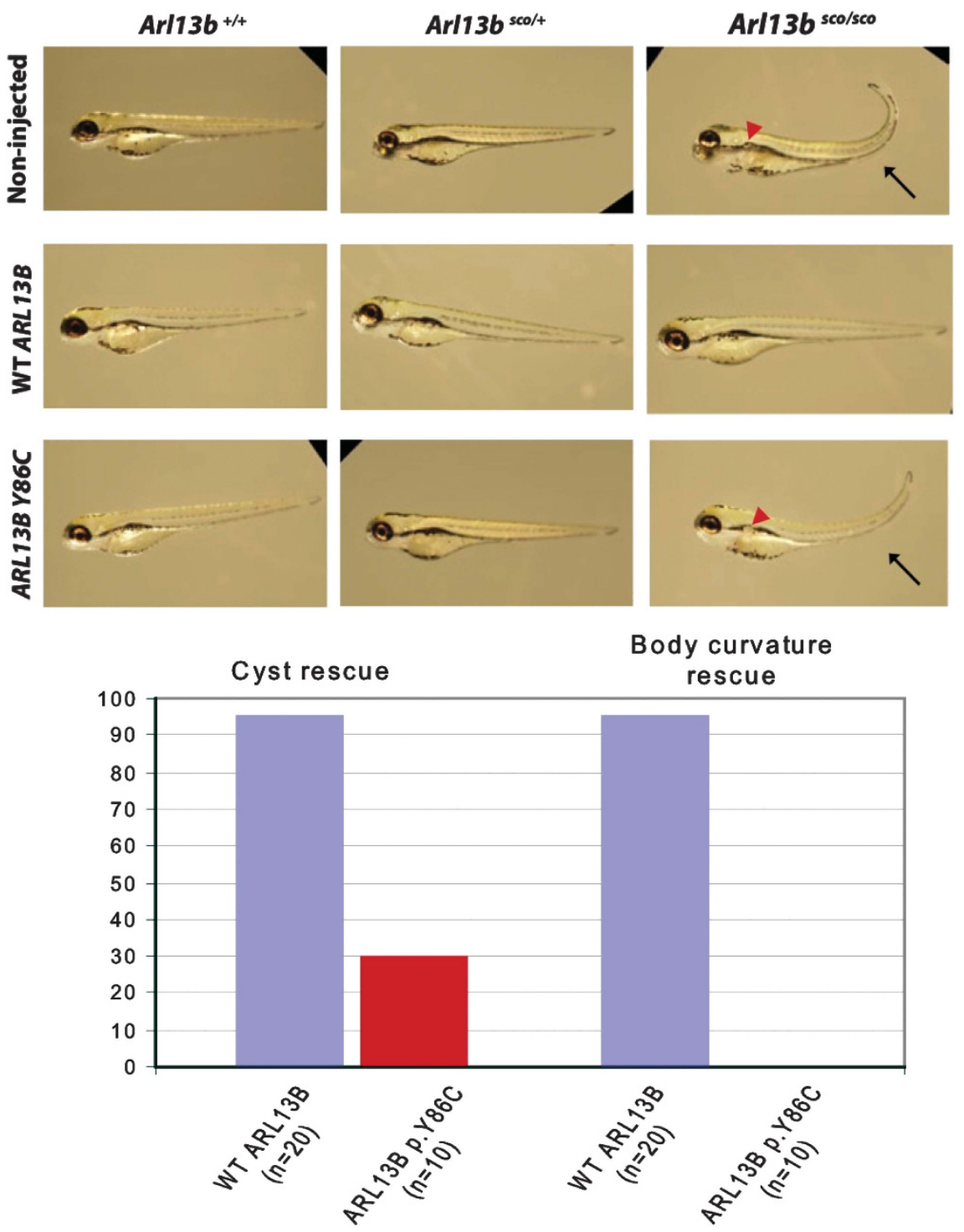

Figure 3 Human wild-type but not p.Y86C-mutated ARL13B rescue the zebrafish scorpion mutant, indicating a hypomorphic allele. Non-injected arl $13 b^{\text {sco/sco }}$ embryos have curved tails (black arrow) and cystic kidney (red arrow). About $95 \%$ of arl13b $b^{\text {sco/sco }}$ embryos injected with human wild-type $A R L 13 B$ RNA show rescue of curved tail and absence of cystic kidney. The human Y86C mRNA showed complete inability to rescue the tail phenotype and significantly decreased ability to rescue the kidney phenotype $(P<0.005$; Fisher's Exact Test).

To validate the functional damaging effect of this $\mathrm{Y} 86 \mathrm{C}$ variant in vivo, we took advantage of the arlizb $b^{s c o}$ zebrafish line. Wild-type human ARL13B can rescue the arl13b $b^{s c o}$ phenotype. ${ }^{9}$ We thus tested the ability of human ARL13B synthetic mRNA with the Y86C variant to rescue the $\operatorname{arl} 13 b^{s c o}$ phenotype. We found that mutated mRNA is not able to efficiently rescue the phenotype as compared to WT human ARL13B synthetic mRNA. As shown in Figure 3, mutated mRNA does not rescue the body curvature phenotype. However, the Y86C variant allows partial rescue, as kidney cysts were not visible in $30 \%$ of the injected mutants (Figure 3 ).

We also took advantage of mouse embryonic fibroblasts (MEFs) from the Arll3b null hennin (Arl13b $b^{h n n}$ ) mouse ${ }^{11}$ to test the effects of this variant. About $70 \%$ of wild-type MEFs show Arl13b-positive cilia upon culture in serum-free condition for $24 \mathrm{~h}$. MEFs that lack Arl13b rarely grow cilia $(\sim 20 \%$ ciliated cells after serum starvation $)$ and their cilia are shorter. ${ }^{12}$ We transfected Arl $3 b^{\text {hnn }}$ MEFs with either a wild-type ARL13B construct or the Y86C ARL13B construct, along with a DsRed cotransfection plasmid to allow visualization of the transfected cells. We measured the ability of each construct to rescue normal ARL13B protein expression in the cilia of Arl13b ${ }^{\text {hnn }}$ MEFs. Previous work in migrating interneurons has shown that proper localization of ARL13B to the cilium is critical for the rescue of ARL13B null phenotypes; ${ }^{22}$ therefore, we did not measure transfected cells that may have grown a cilium but failed to exhibit cilia localization of ARL13B. Instead, only transfected (DsRed-positive) cells possessing an ARL13B-positive cilium were considered to show rescue. Across three independent experiments, we counted a total of 135 cells transfected with wild-type $A R L 13 B$, and 62 of them showed rescue (mean percent rescue: 46\%) (Figure 4). Among 118 cells transfected with mutant ARL13B, only 32 showed rescue (mean percent rescue: 22\%) (Figure 4). Therefore, in both zebrafish and MEFs, the Y86C mutant ARL13B is less efficient than wild-type ARL13B at rescuing the null phenotype.

We next analyzed $A R L 13 B$ expression during human embryonic nervous system development by in situ hybridization. ARL13B 

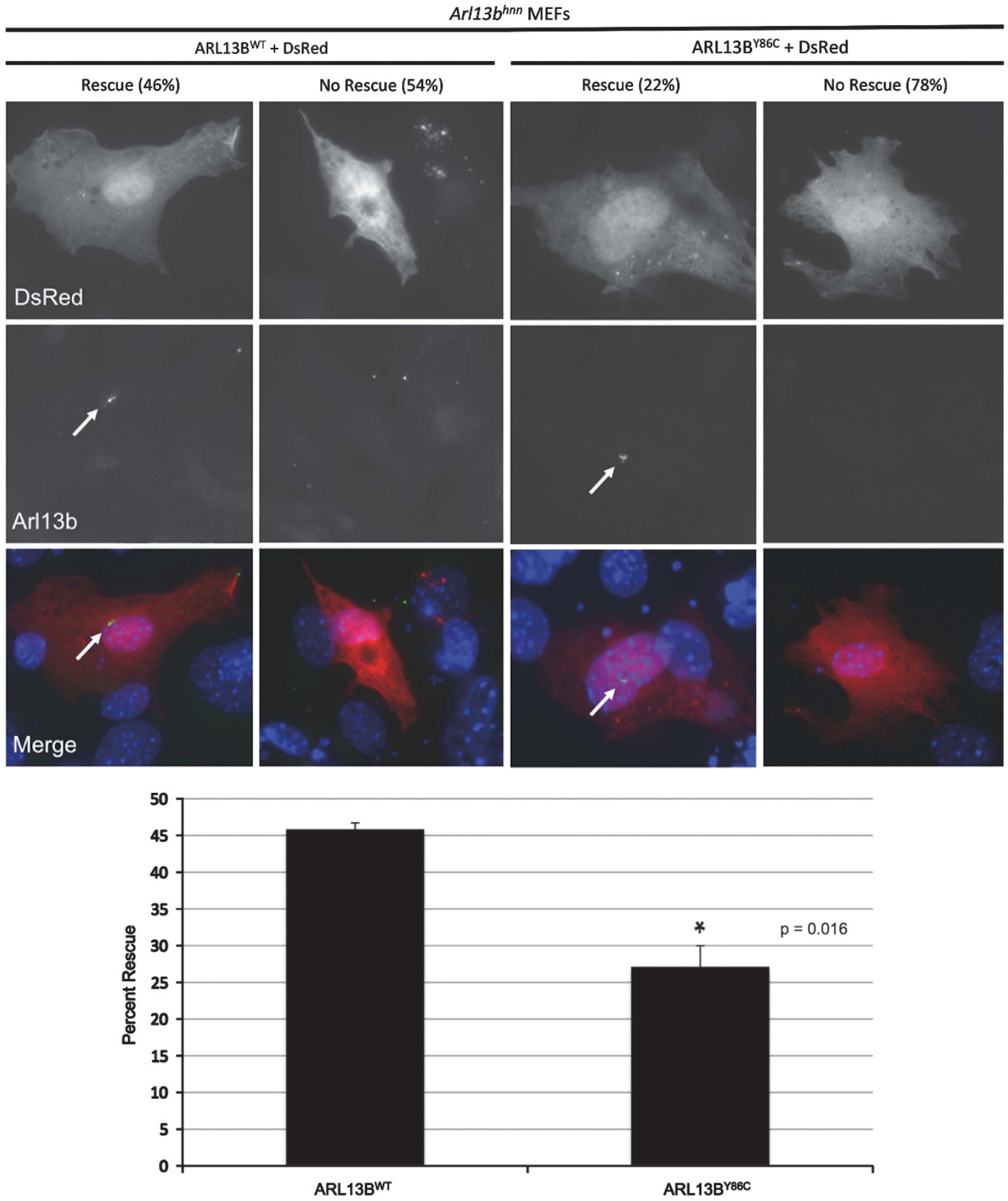

Figure 4 p.Y86C-mutated ARL13B is less efficient at rescuing Arl13bennin (null) mouse embryonic fibroblast (MEF) phenotype. Arl13b $b^{\text {hnn }}$ MEFs were co-transfected with DsRed and either wild-type or p.Y86C-mutated human ARL13B constructs and then immunostained using Arl13b antibody. We showed that $46 \%$ of $A r l 13 b$ null MEF transfected with wild-type $A R L 13 B$ construct showed ARL13B staining in cilia (rescue), while only $22 \%$ of those transfected with mutant $A R L 13 B$ construct did.

was detected in the alar and basal plate of the myelencephalon, as well as in the mesencephalon and metencephalon at CS16 (Figure 5a and b). At CS19, ARL13B is expressed in the ventricular layer of the diencephalon and myelencephalon as well as the tegmentum of the pons and the cerebellar rhombic lips (Figure $5 \mathrm{c}$ and $\mathrm{d}$ ). ARL13B transcript is also found in dorsal root ganglia, the vestibular ganglion and within the neuronal epithelium surrounding the otic vesicle (Figure $5 \mathrm{e}$ and $\mathrm{f}$ ). Because the patient studied presented with obesity, we wondered whether obesity could be linked to ARL13B dysfunction within primary cilia of the developing hypothalamic neurons. Using immunohistochemistry on newborn GFP-centrin transgenic mouse brain, we found that Arl13b is localized within the primary cilia of ventromedial hypothalamic neurons (Figure $5 \mathrm{j}$ and $\mathrm{k}$ ).

\section{DISCUSSION}

We identified a novel missense variant in the $A R L 13 B$ gene in a patient with JS, confirming $A R L 13 B$ as the 8th JS locus (JBTS8). In addition to JS, this patient presented with retinal anomalies and obesity. To date, only two families with $A R L 13 B$ variants have been 

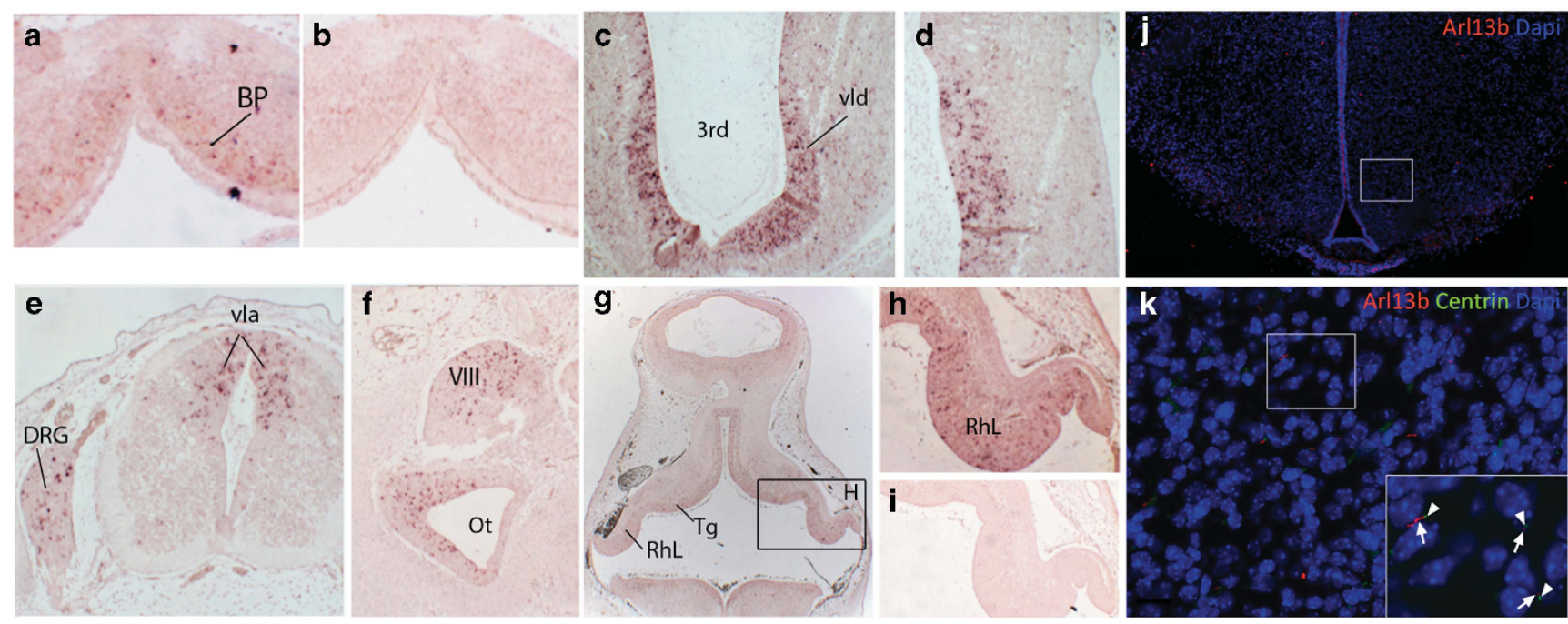

Figure 5 ARL13B expression during development (ISH in humans and IF in mouse). In situ hybridization on CS16 (a, b) and CS19 (C-I) human embryo sections with $A R L 13 B$ antisense probe (a, $\mathbf{c}-\mathbf{h})$ or $A R L 13 B$ sense probe (b, i). ARL13B expression in the basal plate of myelencephalon (a). ARL13B expression in the ventricular layer of diencephalon (c, d), in the ventricular layer of the alar plate of myelencephalon and in dorsal root ganglia (e). $A R L 13 B$ is also expressed in the epithelia surrounding the cochlea, in vestibular ganglion (f) and in pons and cerebellar rhombic lip (g, h). IF on newborn GFPcentrin transgenic mice using Arl13b antibody and showing Arl13b protein localization all along the ciliary axoneme of ventromedial hypothalamic neurons (j, k). 3rd: Third ventricule, 4th: fourth ventricule, bp: basal plate, Ot: otic vesicle, VIII: vestibulocochlear ganglion; RhL: cerebellar rhombic lip; Tg: tegmentum of pons; DRG: dorsal root ganglion; vap: ventricular layer of alar plate, vla: ventricular (ependymal) layer of alar plate of spinal cord, vld: ventricular layer of diencephalon.

reported, ${ }^{9}$ supporting a minor contribution of $A R L 13 B$ to JS. In this previous study, no patient had renal involvement, and one patient displayed evidence of mild nonspecific pigmentary retinopathy on clinical examination, although electroretinogram was normal in the affected siblings, and therefore $A R L 13 B$ involvement in retinal function was not clear. The $\operatorname{Arl} 13 b^{\mathrm{hnn}}$ mouse embryo also displays abnormal eyes, ${ }^{11}$ thus exploring the mouse eye phenotype more precisely should give insight into the role of ARL13B in the development of the retina. In addition, our patient also presented with obesity, which is rarely associated to JS. Observation from one patient is insufficient to argue that $A R L 13 B$ variants may be associated with obesity; however, for other ciliopathies associated to obesity such as ALS, MORM and BBS, one of the causal genes (BBS3) encodes another Arl protein, ARL6. ${ }^{23}$ In addition, we find ARL13B localization within the primary cilia of hypothalamic neurons, especially in the ventromedial nucleus of the hypothalamus, which is considered as a crucial site of regulation of energy homeostasis. ${ }^{24-27}$ Finally, consistent with this finding, recent evidence in ciliopathies has linked obesity to the regulation of homeostasis within the hypothalamus. $^{28}$

Our zebrafish and MEF experiments indicate that the Y86C missense variant identified in this study is hypomorphic, as overexpression of Y86C cDNA is not efficient in rescuing the $\operatorname{arll} 3 b^{s c o}$ zebrafish and Arl13b ${ }^{h n n}$ MEF phenotypes. The early lethality of the Arl13b $b^{h n n}$ mouse indicates a critical role for $A R L 13 B$ in early embryonic development. This was confirmed by our observation of $A R L 13 B$ expression early during human embryonic development and may provide one explanation for the low percentage of $A R L 13 B$ variants in JS cohorts and the absence of homozygous truncating variants reported thus far. Despite two patients displaying an occipital encephalocele, no $A R L 13 B$ variants have been found in Meckel syndrome fetuses (Cantagrel et $a l^{9}$ and personal data), although Meckel syndrome has been shown to be the extreme lethal phenotype of JS for other genes. ${ }^{29-33}$
In conclusion, we have identified a novel homozygous missense variant in ARL13B/JBTS8 in a JS patient with retinal involvement and obesity. We have shown that this variant is hypomorphic, as it is unable to rescue efficiently either the $\operatorname{arl} 13 b^{s c o}$ zebrafish phenotype or the deficiencies in $A r l 13 b^{h n n}$ MEFs. This variant is the first novel $A R L 13 B$ variant reported since the original publication by Cantagrel et al. ${ }^{9}$ reporting three variants in two families. Thus, these results confirm the involvement of $A R L 13 B$ in JS with retinopathy and suggest the extension of the phenotypic spectrum of $A R L 13 B$ variants to obesity.

\section{CONFLICT OF INTEREST}

The authors declare no conflict of interest.

\section{ACKNOWLEDGEMENTS}

We thank the patients and their families for participation. This work and ST were supported by grants from ANR 2010 FOETOCILPATH No. 112201. VC was supported by grant ANR-12-PDOC-0026.

1 Joubert M, Eisenring JJ, Robb JP, Andermann F: Familial agenesis of the cerebellar vermis. A syndrome of episodic hyperpnea, abnormal eye movements, ataxia, and retardation. Neurology 1969; 19: 813-825.

2 Valente EM, Dallapiccola B, Bertini E: Joubert syndrome and related disorders. Handb Clin Neurol 2013; 113: 1879-1888.

3 Badano JL, Mitsuma N, Beales PL, Katsanis N: The ciliopathies: an emerging class of human genetic disorders. Annu Rev Genomics Hum Genet 2006; 7: 125-148.

4 Millen KJ, Gleeson JG: Cerebellar development and disease. Curr Opin Neurobiol 2008; 18: 12-19.

5 Huangfu D, Liu A, Rakeman AS, Murcia NS, Niswander L, Anderson KV: Hedgehog signalling in the mouse requires intraflagellar transport proteins. Nature 2003; 426: 83-87.

6 Chizhikov VV, Davenport J, Zhang Q et al: Cilia proteins control cerebellar morphogenesis by promoting expansion of the granule progenitor pool. J Neurosci Off J Soc Neurosci 2007; 27: 9780-9789.

7 Spassky N, Han Y-G, Aguilar A et al: Primary cilia are required for cerebellar development and Shh-dependent expansion of progenitor pool. Dev Biol 2008; 317: 246-259. 
8 Aguilar A, Meunier A, Strehl L et al: Analysis of human samples reveals impaired SHHdependent cerebellar development in Joubert syndrome/Meckel syndrome. Proc Nat Acad Sci USA 2012; 109: 16951-16956.

9 Cantagrel V, Silhavy JL, Bielas SL et al: Mutations in the cilia gene ARL13B lead to the classical form of Joubert syndrome. Am J Hum Genet 2008; 83: 170-179.

10 Sun Z, Amsterdam A, Pazour GJ, Cole DG, Miller MS, Hopkins N: A genetic screen in zebrafish identifies cilia genes as a principal cause of cystic kidney. Dev Camb Eng/ 2004; 131: 4085-4093.

11 Caspary T, Larkins CE, Anderson KV: The graded response to Sonic Hedgehog depends on cilia architecture. Dev Cell 2007; 12: 767-778.

12 Larkins CE, Aviles GDG, East MP, Kahn RA, Caspary T: Arl13b regulates ciliogenesis and the dynamic localization of Shh signaling proteins. Mol Biol Cell 2011; 22: 4694-4703.

13 Cevik S, Hori Y, Kaplan Ol et al: Joubert syndrome Arl13b functions at ciliary membranes and stabilizes protein transport in Caenorhabditis elegans. J Cell Biol 2010; 188: 953-969.

14 Li Y, Wei Q, Zhang Y, Ling K, Hu J: The small GTPases ARL-13 and ARL-3 coordinate intraflagellar transport and ciliogenesis. J Cell Biol 2010; 189: 1039-1051.

15 Higginbotham $\mathrm{H}$, Guo J, Yokota $\mathrm{Y}$ et al: Arl13b-regulated cilia activities are essential for polarized radial glial scaffold formation. Nat Neurosci 2013; 16: 1000-1007.

16 Romano S, Boddaert N, Desguerre I et al: Molar tooth sign and superior vermian dysplasia: a radiological, clinical, and genetic study. Neuropediatrics 2006; 37 : 42-45.

17 Peitsch MC, Wells TN, Stampf DR, Sussman JL: The Swiss-3DImage collection and PDB-Browser on the World-Wide Web. Trends Biochem Sci 1995; 20 82-84.

18 Kiefer F, Arnold K, Künzli M, Bordoli L, Schwede T: The SWISS-MODEL Repository and associated resources. Nucleic Acids Res 2009; 37: D387-D392.

19 Bullen P, Wilson DI: The Carnegie staging of human embryos: a practical guide; in Strachan T, Lindsay S, Wilson DI (eds); Molecular Genetics of Early Human Development. London: Bios.

20 Cheng Y-Z, Eley L, Hynes A-M et al: Investigating embryonic expression patterns and evolution of AHI1 and CEP290 genes, implicated in Joubert syndrome. PloS One 2012; 7: e44975.
21 Miertzschke M, Koerner C, Spoerner M, Wittinghofer A: Structural insights into the small G-protein Arl13B and implications for Joubert syndrome. Biochem J 2014; 457: 301-311.

22 Higginbotham H, Eom T-Y, Mariani LE et al: Arl13b in primary cilia regulates the migration and placement of interneurons in the developing cerebral cortex. Dev Cell 2012; 23: 925-938.

23 Chiang AP, Nishimura D, Searby $C$ et al: Comparative genomic analysis identifies an ADP-ribosylation factor-like gene as the cause of Bardet-Biedl syndrome (BBS3). Am J Hum Genet 2004; 75: 475-484.

24 Dhillon H, Zigman JM, Ye C et al: Leptin directly activates SF1 neurons in the VMH, and this action by leptin is required for normal body-weight homeostasis. Neuron 2006; 49: 191-203.

25 Hetherington AW, Ranson SW: Nutrition Classics. The Anatomical Record, Volume 78, 1940: Hypothalamic lesions and adiposity in the rat. Nutr Rev 1983; 41: 124-127.

26 King BM: The rise, fall, and resurrection of the ventromedial hypothalamus in the regulation of feeding behavior and body weight. Physiol Behav 2006; 87: 221-244.

27 Klöckener T, Hess S, Belgardt BF et al: High-fat feeding promotes obesity via insulin receptor/PI3K-dependent inhibition of SF-1 VMH neurons. Nat Neurosci 2011; 14: 911-918.

28 Davenport JR, Watts AJ, Roper VC et al: Disruption of intraflagellar transport in adult mice leads to obesity and slow-onset cystic kidney disease. Curr Biol CB 2007; 17: 1586-1594.

29 Baala L, Audollent S, Martinovic J et al: Pleiotropic effects of CEP290 (NPHP6) mutations extend to Meckel syndrome. Am J Hum Genet 2007; 81: 170-179.

30 Delous M, Baala L, Salomon R et al: The ciliary gene RPGRIP1L is mutated in cerebello-oculo-renal syndrome (Joubert syndrome type B) and Meckel syndrome. Nat Genet 2007; 39: 875-881.

31 Baala L, Romano S, Khaddour R et al: The Meckel-Gruber syndrome gene, MKS3, is mutated in Joubert syndrome. Am J Hum Genet 2007; 80: 186-194.

32 Mougou-Zerelli S, Thomas S, Szenker E et al: CC2D2A mutations in Meckel and Joubert syndromes indicate a genotype-phenotype correlation. Hum Mutat 2009; 30: $1574-1582$.

33 Valente EM, Logan CV, Mougou-Zerelli S et al: Mutations in TMEM216 perturb ciliogenesis and cause Joubert, Meckel and related syndromes. Nat Genet 2010; 42: 619-625.

Supplementary Information accompanies this paper on European Journal of Human Genetics website (http://www.nature.com/ejhg) 